\title{
MicroRNA Expression Signature in Human Calcific Aortic Valve Disease
}

\author{
Hui Wang, ${ }^{1}$ Jing Shi, ${ }^{1}$ Beibei Li, ${ }^{1}$ Qiulian Zhou, ${ }^{2}$ Xiangqing Kong, ${ }^{1}$ and Yihua Bei ${ }^{2}$ \\ ${ }^{1}$ Department of Cardiology, The First Affiliated Hospital of Nanjing Medical University, Nanjing 210029, China \\ ${ }^{2}$ Cardiac Regeneration and Ageing Lab, School of Life Science, Shanghai University, Shanghai 200444, China
}

Correspondence should be addressed to Xiangqing Kong; xiangqingkong_nj@163.com and Yihua Bei; yihuabei_sh@163.com

Received 12 September 2016; Accepted 13 February 2017; Published 11 April 2017

Academic Editor: Giovanni Mariscalco

Copyright (C) 2017 Hui Wang et al. This is an open access article distributed under the Creative Commons Attribution License, which permits unrestricted use, distribution, and reproduction in any medium, provided the original work is properly cited.

\begin{abstract}
Altered microRNA (miRNA, miR) expression has been related to many disease processes; however, the miRNA expression signature in calcific aortic valve disease (CAVD) is unclear. In this study, microarrays were used to determine the miRNA expression signature of tissue samples from healthy individuals $(n=4)$ and patients with CAVD $(n=4)$. TargetScan, PITA, and microRNAorg 3way databases were used to predict the potential target genes. DIANA-miRPath was used to incorporate the aberrant miRNAs into gene pathways. miRNA microarrays identified 92 differentially expressed miRNAs in CAVD tissues. The principal component analysis (PCA) of these samples and the unsupervised hierarchical clustering analysis based on the 92 aberrantly expressed miRNAs noted that miRNA expression could be categorized into two well-defined clusters that corresponded to healthy control and CAVD. Bioinformatic analysis showed the miRNA targets and potential molecular pathways. Collectively, our study reported the miRNA expression signature in CAVD and may provide potential therapeutic targets for CAVD.
\end{abstract}

\section{Background}

Valve diseases continue to occur in many patients with significant morbidity and mortality. The age-adjusted prevalence of moderate or severe valve diseases was estimated at $2.5 \%$ [1]. Calcific aortic valve disease (CAVD) is the most common valve heart disease in the elderly and a leading cause of aortic stenosis [2]. In developing countries, CAVD represents a major cause for surgical valve replacement [3]. As a result of rising life expectancy and ageing populations, the burden of CAVD will significantly increase in the near future.

While CAVD was originally thought to be a degenerative process with passive deposition of calcium phosphate in the valve occurring with age, it now appears to be a complex and actively regulated progress mediated by inflammation, cell apoptosis, lipid deposition, renin-angiotensin system activation, extracellular matrix remodeling, and bone formation [4-6]. To better monitor progression of CAVD and identify the most appropriate instances for surgical intervention, biomarkers can be serially monitored. Such biomarkers would represent objective laboratory measurements, as older patients with CAVD might have atypical symptoms associated with comorbidities such as pulmonary disease or orthopaedic disabilities $[7,8]$.

MicroRNAs (miRNAs, miRs) are endogenous, small, single-stranded, 21-25 nucleotide noncoding RNAs, regulating target gene expressions by hybridizing to messenger RNAs (mRNAs). An individual miRNA is able to target tens to hundreds of genes while a single gene can also be targeted by lots of miRNAs [9]. Since miRNAs play critical roles in many physiological processes, increasing reports indicate that a distinct pattern of altered miRNA expressions may be linked to specific disease processes [10-15]. We previously reported the miRNA expression signature in degenerative aortic stenosis [14]. In this study, we explored the miRNA expression signature in CAVD.

\section{Materials and Methods}

2.1. Tissue Sample Collection and RNA Isolation. This study was officially approved by the Ethics Committees of the First Affiliated Hospital of Nanjing Medical University and conformed to the principles outlined in the Declaration of Helsinki. All written informed consent was obtained 
from patients, and parents where applicable. Tissue samples from four healthy subjects were collected from prospective multiorgan donors in cases because of technical reasons that prevented transplantation, while stenotic aortic valve samples were obtained from four patients who underwent surgical valve replacement for aortic stenosis. All samples were examined by gross examination, and microscopic examination of hematoxylin and eosin-stained cryosections was conducted to confirm the presence/absence of CAVD. Tissue samples harvested from subject donors were snap-frozen in liquid nitrogen, and RNA was then isolated using the RNeasy Mini Kit (Qiagen, Hilden, Germany) according to the manufacturer's instructions.

2.2. miRNA Microarray Analysis. Total RNAs were isolated from heart tissues using mirVana ${ }^{\mathrm{TM}}$ RNA Isolation Kit, quantified by NanoDrop ND-2100 (Thermo Scientific), and controlled for RNA integrity using Agilent Bioanalyzer 2100 (Agilent Technologies) according to the manufacturer's instructions. miRNA profiling was performed with $\mathrm{OE}$ Biotech's (Shanghai, China) miRNA microarray service. The arrays from the control group are the same as we previously used [14].

2.3. Bioinformatic Analysis. TargetScan, PITA, and microRNAorg 3-way databases were used to identify potential human miRNA target genes and a Venn diagram was made to provide relations among the 3 databases. DIANA tool miRPath v2.0, a web-based analysis tool, was used for pathway enrichment analysis for the miRNA set identified [16]. DIANA tool miRPath assigns Kyoto Encyclopedia of Genes and Genomes (KEGG) pathway with the significance level determined by the number of target genes affected by the identified microRNAs.

2.4. Statistical Analysis. Independent Student's $t$-test was used to determine whether there were any significant differences between the miRNA expression profiles between two groups. $P$ values less than $0.05(P<0.05)$ were considered to be statistically significant. Significant data were further analyzed by clustering, and the expression profiles were visualized with GeneSpring 10.0 (Agilent Technology).

\section{Results}

3.1. Principal Component Analysis of miRNA Expression Profiles. Principal component analysis (PCA) is a mathematical algorithm that reduces the dimensionality of the data while retaining most of the variation in the data set [17]. Dots in two colors separated in two axes based on the differences of the data, suggesting that samples in this study were prepared appropriately and could be grouped as CAVD or healthy control (Figure 1).

3.2. Unsupervised Hierarchical Cluster Analysis of miRNA Microarray Data. miRNA arrays identified 92 miRNAs with a statistically significant differential expression of 2.0 -fold or greater in CAVD samples relative to normal controls.

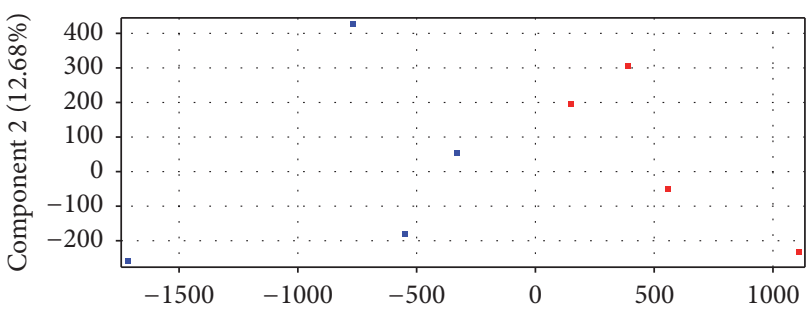

Figure 1: PCA of the miRNA profiles in CAVD tissue samples and control subjects. Red dots represent samples from control group, while blue dots represent samples from CAVD group.

Fifty-three miRNAs were underexpressed and 39 were overexpressed in aortic tissue from CAVD patients (Table 1). Unsupervised hierarchic clustering of the two groups was performed on the 92 differently expressed miRNAs and displayed as heatmap (Figure 2).

3.3. Target Genes Analysis. MicroRNAorg, TargetScan, and PITA were used to predict the targets of differentially expressed miRNAs in CAVD samples. A Venn diagram was made to highlight the relations among the three databases. There are 8717 genes overlapping by all three sets, which are most likely to be targets of miRNAs in patients with CAVD (Figure 3).

3.4. DIANA miRNA Pathway Analysis. To better understand the putative mechanisms underlying CAVD, we used DIANA-miRPath (v2.0), a web-based server developed to identify the potential cellular pathways regulated by microRNAs. We first evaluated downregulated miRNAs in CAVD samples compared to control samples. The potential affected pathways included the following: cell cycle, PI3K-Akt signaling pathway, ECM-receptor interaction, HIF-1 signaling pathway, p53 signaling pathway, ErbB signaling pathway, Neurotrophin signaling pathway, focal adhesion, and DNA replication (Table 2). Upregulated miRNAs were also used to generate the potential affected pathways by DIANA-miRPath and identified p53 signaling pathway, HIF-1 signaling pathway, valine, leucine, and isoleucine biosynthesis, ErbB signaling pathway, cell cycle, mTOR signaling pathway, MAPK signaling pathway, PI3K-Akt signaling pathway, Wnt signaling pathway, synthesis and degradation of ketone bodies, TGF-beta signaling pathway, basal transcription factors, glycerophospholipid metabolism, hypertrophic cardiomyopathy (HCM), focal adhesion, circadian rhythm, mismatch repair, lysine degradation, and butanoate metabolism (Table 3).

\section{Discussion}

miRNAs have been shown to be critical regulators in cardiovascular diseases [18-25]. However, there are no reports revealing distinct miRNA expression signatures in the CAVD patients and healthy controls. In this study, we identified global changes in the miRNA expression profile in CAVD and healthy control. Calcific aortic valve stenosis is characterized by lipid accumulation, inflammation, formation 
TABLE 1: Fifty-three underexpressed and 39 overexpressed miRNAs in aortic tissue from CAVD patients compared to the control group.

\begin{tabular}{|c|c|c|c|}
\hline Systematic name & $P$ value & Fold change & Regulation \\
\hline hsa-miR-3656 & 0.011202 & 2.0143232 & $\mathrm{Up}$ \\
\hline hsa-miR-765 & 0.033639 & 2.0401733 & Up \\
\hline hsa-miR-2861 & 0.008741 & 2.0782373 & Up \\
\hline hsa-miR-663a & 0.00633 & 2.0819619 & Up \\
\hline hsa-miR-1246 & 0.027045 & 2.1549542 & Up \\
\hline hsa-miR-3141 & 0.002969 & 2.2772849 & Up \\
\hline hsa-miR-125a-3p & 0.012002 & 2.3428166 & Up \\
\hline hsa-miR-4327 & 0.004807 & 2.367507 & Up \\
\hline hsa-miR-638 & 0.002785 & 2.3686504 & Up \\
\hline hsa-miR-4270 & 0.030574 & 2.4421182 & Up \\
\hline hsa-miR-642b-3p & 0.034575 & 2.5763547 & Up \\
\hline hsa-miR-513a-5p & 0.007538 & 2.6546612 & Up \\
\hline hsa-miR-483-5p & 0.008099 & 2.655595 & Up \\
\hline hsa-miR-3679-5p & 0.004016 & 2.7413363 & Up \\
\hline hsa-miR-3648 & 0.017053 & 2.8097122 & Up \\
\hline hsa-miR-30c-1-3p & $7.64 \mathrm{E}-04$ & 2.893416 & Up \\
\hline hsa-miR-1275 & 0.005457 & 2.9556682 & Up \\
\hline hsa-miR-513b & 0.020604 & 3.4257321 & Up \\
\hline hsa-miR-1972 & 0.011389 & 3.689852 & Up \\
\hline hsa-miR-3138 & 0.004076 & 3.974949 & Up \\
\hline hsa-miR-3663-3p & 0.005874 & 4.314916 & Up \\
\hline hsa-miR-21-5p & 0.003826 & 4.317176 & Up \\
\hline hsa-miR-718 & 0.031718 & 4.957687 & Up \\
\hline hsa-miR-630 & 0.001304 & 7.3841376 & Up \\
\hline hsa-miR-575 & 0.001586 & 10.079804 & Up \\
\hline hsa-miR-3934 & 0.048827 & 13.458452 & Up \\
\hline hsa-miR-143-5p & 0.033632 & 16.2909 & Up \\
\hline hsa-miR-3131 & 0.047501 & 22.065104 & Up \\
\hline hsa-miR-125b-1-3p & 0.00519 & 23.412596 & Up \\
\hline hsa-miR-625-3p & 0.005867 & 23.648891 & Up \\
\hline hsa-miR-1471 & 0.035145 & 25.31259 & Up \\
\hline hsa-miR-4314 & 0.032125 & 27.92129 & Up \\
\hline hsa-miR-636 & 0.00923 & 28.36478 & Up \\
\hline hsa-miR-3945 & 0.018336 & 38.864952 & Up \\
\hline hsa-miR-3610 & 0.014352 & 49.196358 & Up \\
\hline hsa-miR-1182 & $1.46 \mathrm{E}-05$ & 73.30232 & Up \\
\hline hsa-miR-3713 & $2.44 \mathrm{E}-05$ & 117.90961 & Up \\
\hline hsa-miR-21-3p & 5.95E-05 & 149.08258 & Up \\
\hline hsa-miR-516a-5p & $2.88 \mathrm{E}-05$ & 155.79349 & $\mathrm{Up}$ \\
\hline hsa-miR-654-3p & 0.002559 & 2.0230756 & Down \\
\hline hsa-miR-93-5p & 0.020279 & 2.0238087 & Down \\
\hline hsa-miR-320d & 0.038247 & 2.052202 & Down \\
\hline hsa-miR-381 & 0.035566 & 2.0584567 & Down \\
\hline hsa-miR-214-3p & 0.011756 & 2.0957778 & Down \\
\hline hsa-miR-125b-5p & 0.036771 & 2.1063256 & Down \\
\hline hsa-miR-361-3p & 0.036059 & 2.1256602 & Down \\
\hline hsa-miR-29c-3p & 0.009743 & 2.1358023 & Down \\
\hline hsa-miR-495 & 0.001289 & 2.1425073 & Down \\
\hline hsa-miR-374a-5p & 0.01904 & 2.1439137 & Down \\
\hline hsa-miR-20b-5p & 0.027368 & 2.1694279 & Down \\
\hline hsa-miR-382-5p & 0.043289 & 2.1756916 & Down \\
\hline
\end{tabular}

TABLE 1: Continued.

\begin{tabular}{lccc}
\hline Systematic name & $P$ value & Fold change & Regulation \\
\hline hsa-miR-4324 & 0.039752 & 2.178068 & Down \\
hsa-miR-25-3p & 0.004579 & 2.1911306 & Down \\
hsa-miR-100-5p & 0.035509 & 2.1913323 & Down \\
hsa-miR-193b-3p & 0.022676 & 2.191656 & Down \\
hsa-miR-107 & 0.007782 & 2.2016506 & Down \\
hsa-miR-660-5p & $8.68 \mathrm{E}-04$ & 2.2094207 & Down \\
hsa-miR-103a-3p & 0.020178 & 2.2370007 & Down \\
hsa-miR-195-5p & 0.049708 & 2.3292358 & Down \\
hsa-miR-299-5p & 0.002066 & 2.3574395 & Down \\
hsa-miR-487b & $7.71 E-04$ & 2.422462 & Down \\
hsa-miR-128 & 0.022531 & 2.4691415 & Down \\
hsa-miR-181d & 0.019566 & 2.5412066 & Down \\
hsa-miR-374b-5p & 0.038373 & 2.5474217 & Down \\
hsa-let-7b-5p & 0.042981 & 2.6197023 & Down \\
hsa-miR-140-5p & 0.005972 & 2.639281 & Down \\
hsa-let-7g-5p & 0.02818 & 2.7145965 & Down \\
hsa-miR-15la-5p & 0.010444 & 2.7681546 & Down \\
hsa-miR-532-5p & 0.020008 & 2.7994845 & Down \\
hsa-miR-26b-5p & 0.025414 & 2.8088717 & Down \\
hsa-miR-30e-3p & 0.020305 & 2.8739653 & Down \\
hsa-miR-140-3p & 0.040144 & 2.8943768 & Down \\
hsa-miR-29c-5p & 0.004665 & 2.9975233 & Down \\
hsa-miR-181c-5p & 0.015322 & 3.0353231 & Down \\
hsa-miR-204-5p & 0.044941 & 3.0471704 & Down \\
hsa-let-7d-5p & 0.025658 & 3.0987353 & Down \\
hsa-miR-98 & 0.027495 & 3.2012997 & Down \\
hsa-miR-10a-5p & 0.019225 & 3.3482268 & Down \\
hsa-miR-505-5p & $1.18 \mathrm{E}-05$ & 42.592148 & Down \\
hsa-let-7f-5p & 0.037282 & 3.4332418 & Down \\
hsa-let-7e-5p & 0.018337 & 3.6489105 & Down \\
hsa-let-7a-5p & 0.029403 & 3.6912477 & Down \\
hsa-miR-99a-5p & 0.035923 & 3.8172672 & Down \\
hsa-let-7c & 0.032672 & 3.9863558 & Down \\
hsa-miR-126-3p & 0.026073 & 4.528461 & Down \\
hsa-miR-29b-1-5p & 0.006566 & 15.589992 & Down \\
hsa-miR-181c-3p & 0.008219 & 16.225191 & Down \\
\hline & 0.011511 & 20.940771 & Down \\
hsa-miR-194-5p & $1.60 \mathrm{E}-06$ & 70.24472 & Down \\
\hline
\end{tabular}

of plaque neovessels, hemorrhages, neointimal formation, vascular fibrosis, and ectopic calcification [4, 26]. Previous studies have shown that miRNAs play crucial roles in those processes such as angiogenesis, fibrogenesis, proliferation, and apoptosis [9].

miR-126 is one of the most abundantly expressed microRNAs in endothelial cells (ECs) [27]. Upregulation of miR-126 increases EC survival, decreases EC apoptosis, and prevents reactive oxygen species (ROS) mediated endothelial damage 
TABLE 2: The pathways incorporated by downregulated microRNAs in CAVD.

\begin{tabular}{lccc}
\hline KEGG pathway & $P$ value & Genes & miRNAs \\
\hline Cell cycle & $<1 E-16$ & 48 & 19 \\
PI3K-Akt signaling pathway & $2.22 E-16$ & 95 & 9 \\
ECM-receptor interaction & $1.85 E-12$ & 30 & 4 \\
HIF-1 signaling pathway & $8.63 E-10$ & 28 & 15 \\
p53 signaling pathway & $1.49 E-08$ & 23 & 14 \\
ErbB signaling pathway & $7.14 E-05$ & 19 & 10 \\
Neurotrophin signaling pathway & 0.00249 & 19 & 6 \\
Focal adhesion & 0.0029 & 19 & 2 \\
DNA replication & 0.013417 & & \\
\hline
\end{tabular}

TABLE 3: The pathways affected by upregulation of specific microRNAs in CAVD.

\begin{tabular}{|c|c|c|c|}
\hline KEGG pathway & $P$ value & Genes & miRNAs \\
\hline p53 signaling pathway & $5.63 E-09$ & 11 & 2 \\
\hline HIF-1 signaling pathway & 0.00023 & 10 & 2 \\
\hline Valine, leucine, and isoleucine biosynthesis & 0.00227 & 1 & 1 \\
\hline ErbB signaling pathway & 0.003959 & 5 & 1 \\
\hline Cell cycle & 0.003959 & 10 & 2 \\
\hline mTOR signaling pathway & 0.004909 & 6 & 1 \\
\hline MAPK signaling pathway & 0.005193 & 15 & 2 \\
\hline PI3K-Akt signaling pathway & 0.005193 & 17 & 2 \\
\hline Wnt signaling pathway & 0.008586 & 10 & 1 \\
\hline Synthesis and degradation of ketone bodies & 0.009366 & 2 & 1 \\
\hline TGF-beta signaling pathway & 0.021216 & 7 & 1 \\
\hline Basal transcription factors & 0.029417 & 4 & 1 \\
\hline Glycerophospholipid metabolism & 0.030271 & 8 & 1 \\
\hline Hypertrophic cardiomyopathy (HCM) & 0.030271 & 6 & 1 \\
\hline Focal adhesion & 0.030271 & 11 & 2 \\
\hline Circadian rhythm & 0.030848 & 3 & 1 \\
\hline Mismatch repair & 0.034518 & 2 & 1 \\
\hline Lysine degradation & 0.038471 & 4 & 1 \\
\hline Butanoate metabolism & 0.038615 & 3 & 1 \\
\hline
\end{tabular}

Specific types of cancers and infections were not included.

[28]. Our findings of decreased miR-126 in CAVD may suggest a detrimental effect in human calcific aortic valve.

The differentially expressed miRNAs identified in the current study also included many profibrotic miRNAs such as miR-21 and miR-125b that might contribute to CAVD by promoting fibrosis. Several expression profiling studies identify that increased level of miR-21-5p in cardiac fibroblasts promotes cardiac fibrosis via its target genes: phosphatase and tensin homolog (PTEN) [29] and Sprouty-1 (Spry1) [30]. Additionally, miR-125b is a novel regulator of cardiac fibrogenesis, proliferation, and fibroblast-to-myofibroblast transition. Nagpal et al. demonstrated the upregulation of miR-125b in fibrotic human heart and murine models of cardiac fibrosis [31].

Interestingly, our miRNA array data revealed that several members of the let- 7 (let- $7 a$, let-7b, let-7c, let-7d, let-7e, let$7 \mathrm{f}$, and let-7g) were downregulated in calcific aortic valve.
Let-7g targets the genes related to vascular smooth muscle cell (VSMC) functions, including ROS, autophagy-related proteins (expression of beclin-1, LC3-II, and Atg5), and apoptosis-related proteins (expression of caspase-3, Bax, Bcl2 , and $\mathrm{Bcl}-\mathrm{xL}$ ) [32]. Let-7 family members might directly influence aortic valve sclerosis by regulating the proliferation, migration, autophagy, and apoptosis of VSMC, which have been implicated in the progression of CAVD $[4,26]$.

The abnormal expression of miR-21-5p was found in many cardiovascular diseases [33]. Programmed cell death 4 (PDCD4) is identified as a direct target gene of miR-21-5p. It has been reported that miR-21-5p prevented cardiomyocyte apoptosis in ischaemia/reperfusion heart model through PDCD4 repression [34]. Furthermore, miR-21/PDCD4 pathway was proved to be involved in cardiac valvulogenesis by regulating endothelial cell migration [35]. In our work, miR21-5p was upregulated in calcific aortic valve which indicates 


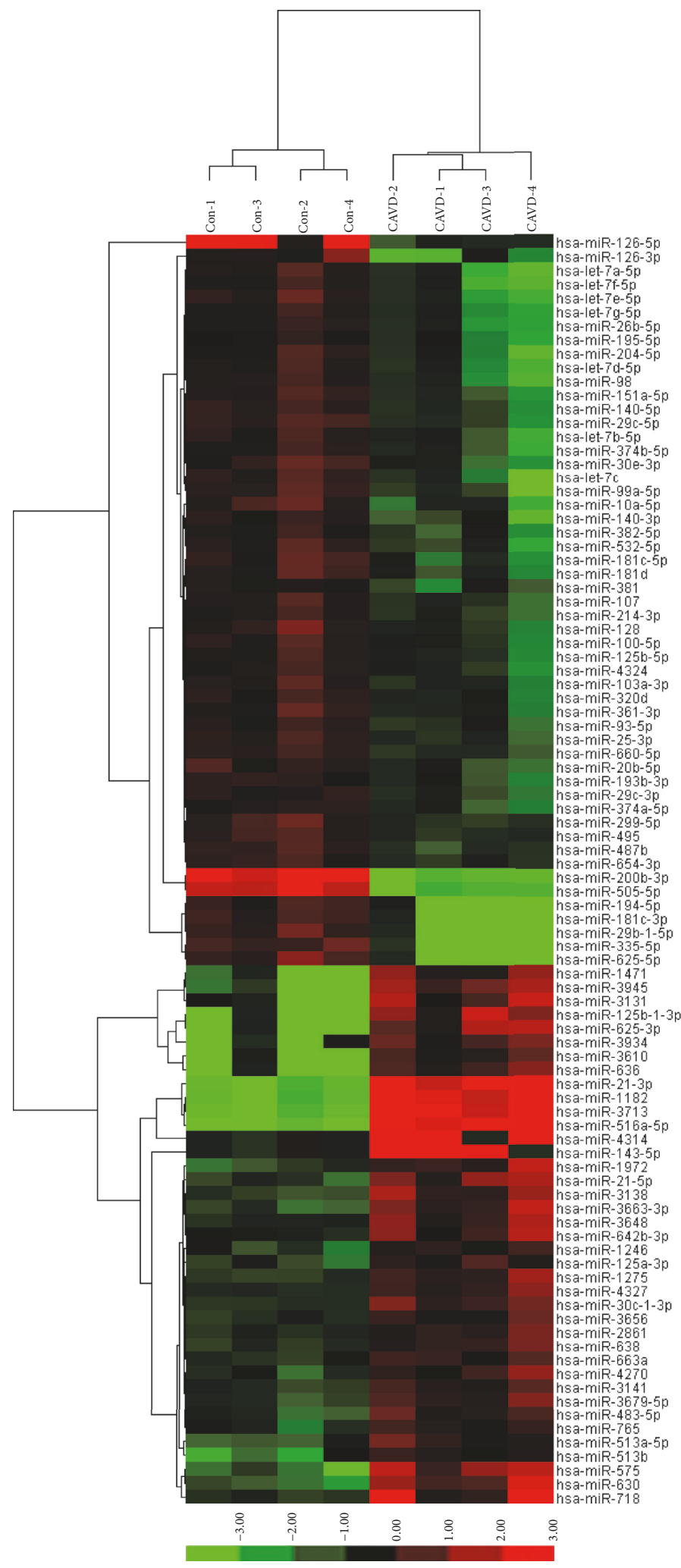

FIGURE 2: Unsupervised hierarchical clustering identified two distinct groups (CAVD versus control) based on their miRNA expression profile. Sample names are listed at top. The names of the significantly altered $(P$ value $<0.05)$ miRNAs are shown at right. Ninety-two miRNAs were expressed differently between the two groups. 


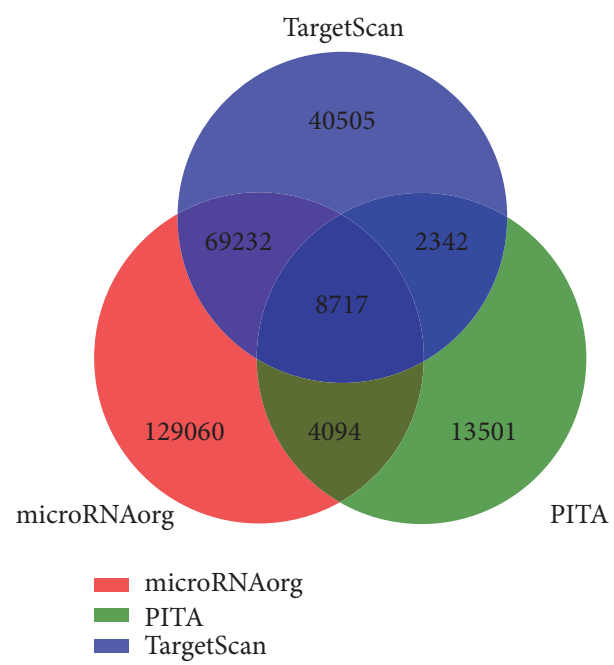

FIGURE 3: The red, green, and blue sets stand for target genes predicted by database microRNAorg, TargetScan, and PITA, respectively.

that miR-21-5p might take part in CAVD. However, the effects and mechanisms of miR-21-5p on calcific aortic valve are still to be investigated in further studies.

A comprehensive knowledge of miRNA expression is essential to improve our understanding of this disease. This study provides the first evidence that there exists a distinct miRNA expression signature in individuals with CAVD, as compared to healthy controls. There are 92 differently expressed miRNAs in the CAVD patients compared with healthy controls by miRNA arrays. PCA and unsupervised hierarchical clustering with these miRNAs demonstrates that this profile could accurately classify the samples according to their disease status. Moreover, bioinformatic tools indicate that the differential expression of miRNAs could be linked to several targets and pathways.

As a limitation of our study, the exact pathways by which dysregulated miRNAs cause CAVD in human remain elusive. Further studies are required to fully characterize the function of candidate miRNAs.

\section{Conclusions}

Taken together, the current study provides insight into the importance of microRNA expression signature in CAVD. A deeper understanding of the molecular alternations in CAVD may provide potential targets for future clinical applications.

\section{Conflicts of Interest}

The authors have declared that no conflicts of interest exist.

\section{Acknowledgments}

This work was supported by the grant from National Natural Science Foundation of China (81400647 to Y. Bei). Dr. X. Kong is a Fellow at the Collaborative Innovation Center For
Cardiovascular Disease Translational Medicine and this work was supported by the grants from the center.

\section{References}

[1] V. T. Nkomo, J. M. Gardin, T. N. Skelton, J. S. Gottdiener, C. G. Scott, and M. Enriquez-Sarano, "Burden of valvular heart diseases: a population-based study," Lancet, vol. 368, no. 9540, pp. 1005-1011, 2006.

[2] B. Iung and A. Vahanian, "Epidemiology of acquired valvular heart disease," Canadian Journal of Cardiology, vol. 30, no. 9, pp. 962-970, 2014.

[3] A. Vahanian, B. Iung, D. Himbert, and P. Nataf, "Changing demographics of valvular heart disease and impact on surgical and transcatheter valve therapies," The International Journal of Cardiovascular Imaging, vol. 27, no. 8, pp. 1115-1122, 2011.

[4] N. M. Rajamannan, F. J. Evans, E. Aikawa et al., "Calcific aortic valve disease: not simply a degenerative process: a review and agenda for research from the national heart and lung and blood institute aortic stenosis working group," Circulation, vol. 124, no. 16, pp. 1783-1791, 2011.

[5] B. Jian, N. Narula, Q.-Y. Li, E. R. Mohler III, and R. J. Levy, "Progression of aortic valve stenosis: TGF- $\beta 1$ is present in calcified aortic valve cusps and promotes aortic valve interstitial cell calcification via apoptosis," The Annals of Thoracic Surgery, vol. 75, no. 2, pp. 457-465, 2003.

[6] A. Galeone, G. Brunetti, A. Oranger et al., "Aortic valvular interstitial cells apoptosis and calcification are mediated by TNF-related apoptosis-inducing ligand," International Journal of Cardiology, vol. 169, no. 4, pp. 296-304, 2013.

[7] J. Bergler-Klein, M. Gyöngyösi, and G. Maurer, "The role of biomarkers in valvular heart disease: focus on natriuretic peptides," The Canadian Journal of Cardiology, vol. 30, no. 9, pp. 1027-1034, 2014.

[8] G. Latsios, D. Tousoulis, E. Androulakis et al., "Monitoring calcific aortic valve disease: the role of biomarkers," Current Medicinal Chemistry, vol. 19, no. 16, pp. 2548-2554, 2012.

[9] J. Shi, Y. Bei, X. Kong et al., "miR-17-3p contributes to exercise-induced cardiac growth and protects against myocardial ischemia-reperfusion injury," Theranostics, vol. 7, no. 3, pp. 664676, 2017.

[10] E. E. Creemers, A. J. Tijsen, and Y. M. Pinto, "Circulating MicroRNAs: novel biomarkers and extracellular communicators in cardiovascular disease?" Circulation Research, vol. 110, no. 3, pp. 483-495, 2012.

[11] H. Wang, Y. Bei, P. Huang et al., "Inhibition of miR-155 protects against LPS-induced cardiac dysfunction and apoptosis in mice," Molecular Therapy-Nucleic Acids, vol. 5, article e374, 2016.

[12] H. Wang, Y. Bei, S. Shen et al., "miR-21-3p controls sepsis-associated cardiac dysfunction via regulating SORBS2," Journal of Molecular and Cellular Cardiology, vol. 94, pp. 43-53, 2016.

[13] L. Tao, Y. Bei, P. Chen et al., "Crucial role of miR-433 in regulating cardiac fibrosis," Theranostics, vol. 6, no. 12, pp. 20682083, 2016.

[14] J. Shi, H. Liu, H. Wang, and X. Kong, "MicroRNA expression signature in degenerative aortic stenosis," BioMed Research International, vol. 2016, Article ID 4682172, 6 pages, 2016.

[15] H. Wang, Y. Bei, J. Shi, J. Xiao, and X. Kong, "Non-coding RNAs in cardiac aging," Cellular Physiology and Biochemistry, vol. 36, no. 5, pp. 1679-1687, 2015. 
[16] I. S. Vlachos, N. Kostoulas, T. Vergoulis et al., "DIANA miRPath v.2.0: investigating the combinatorial effect of microRNAs in pathways," Nucleic Acids Research, vol. 40, no. 1, pp. W498W504, 2012.

[17] M. Ringnér, "What is principal component analysis?" Nature Biotechnology, vol. 26, no. 3, pp. 303-304, 2008.

[18] G. Condorelli, M. V. G. Latronico, and E. Cavarretta, "MicroRNAs in cardiovascular diseases: current knowledge and the road ahead," Journal of the American College of Cardiology, vol. 63, no. 21, pp. 2177-2187, 2014.

[19] X. Liu, J. Xiao, H. Zhu et al., "MiR-222 is necessary for exerciseinduced cardiac growth and protects against pathological cardiac remodeling," Cell Metabolism, vol. 21, no. 4, pp. 584-595, 2015.

[20] D. Lv, J. Liu, C. Zhao et al., "Targeting microRNAs in pathological hypertrophy and cardiac failure," Mini-Reviews in Medicinal Chemistry, vol. 15, no. 6, pp. 475-478, 2015.

[21] J. Xu, Y. Tang, Y. Bei et al., "miR-19b attenuates H2O2-induced apoptosis in rat $\mathrm{H} 9 \mathrm{C} 2$ cardiomyocytes via targeting PTEN," Oncotarget, vol. 7, no. 10, pp. 10870-10878, 2016.

[22] M.-T. Piccoli, C. Bär, and T. Thum, "Non-coding RNAs as modulators of the cardiac fibroblast phenotype," Journal of Molecular and Cellular Cardiology, vol. 92, pp. 75-81, 2016.

[23] Y. F. Melman, R. Shah, and S. Das, "MicroRNAs in heart failure is the picture becoming less miRky?" Circulation: Heart Failure, vol. 7, no. 1, pp. 203-214, 2014.

[24] B. Simonson and S. Das, "MicroRNA therapeutics: the next magic bullet?" Mini-Reviews in Medicinal Chemistry, vol. 15, no. 6, pp. 467-474, 2015.

[25] Z. Lei, J. P. G. Sluijter, and A. van Mil, "MicroRNA therapeutics for cardiac regeneration," Mini-Reviews in Medicinal Chemistry, vol. 15, no. 6, pp. 441-451, 2015.

[26] R. V. Freeman and C. M. Otto, "Spectrum of calcific aortic valve disease: pathogenesis, disease progression, and treatment strategies," Circulation, vol. 111, no. 24, pp. 3316-3326, 2005.

[27] T. Yan, Y. Liu, K. Cui, B. Hu, F. Wang, and L. Zou, "MicroRNA126 regulates EPCs function: implications for a role of miR-126 in preeclampsia," Journal of Cellular Biochemistry, vol. 114, no. 9, pp. 2148-2159, 2013.

[28] S. M. J. Welten, E. A. C. Goossens, P. H. A. Quax, and A. Y. Nossent, "The multifactorial nature of microRNAs in vascular remodelling," Cardiovascular Research, vol. 110, no. 1, pp. 6-22, 2016.

[29] S. Roy, S. Khanna, S.-R. A. Hussain et al., "MicroRNA expression in response to murine myocardial infarction: MiR-21 regulates fibroblast metalloprotease-2 via phosphatase and tensin homologue," Cardiovascular Research, vol. 82, no. 1, pp. 21-29, 2009.

[30] T. Thum, C. Gross, J. Fiedler et al., "MicroRNA-21 contributes to myocardial disease by stimulating MAP kinase signalling in fibroblasts," Nature, vol. 456, no. 7224, pp. 980-984, 2008.

[31] V. Nagpal, R. Rai, A. T. Place et al., "MiR-125b is critical for fibroblast-to-myofibroblast transition and cardiac fibrosis," Circulation, vol. 133, no. 3, pp. 291-301, 2016.

[32] M.-H. Bao, X. Feng, Y.-W. Zhang, X.-Y. Lou, Y. U. Cheng, and H.-H. Zhou, "Let-7 in cardiovascular diseases, heart development and cardiovascular differentiation from stem cells," International Journal of Molecular Sciences, vol. 14, no. 11, pp. 23086-23102, 2013.

[33] Y. Wei, A. Schober, and C. Weber, "Pathogenic arterial remodeling: the good and bad of microRNAs," American Journal of
Physiology-Heart and Circulatory Physiology, vol. 304, no. 8, pp. H1050-H1059, 2013.

[34] Y. Cheng, P. Zhu, J. Yang et al., "Ischaemic preconditioningregulated miR-21 protects heart against ischaemia/reperfusion injury via anti-apoptosis through its target PDCD4," Cardiovascular Research, vol. 87, no. 3, pp. 431-439, 2010.

[35] H. J. Kolpa, D. S. Peal, S. N. Lynch et al., "MiR-21 represses Pdcd4 during cardiac valvulogenesis," Development, vol. 140, no. 10, pp. 2172-2180, 2013. 


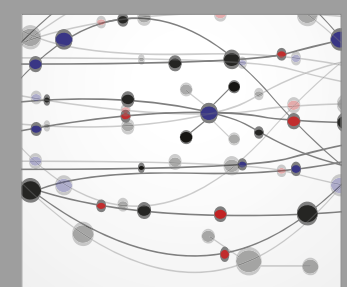

The Scientific World Journal
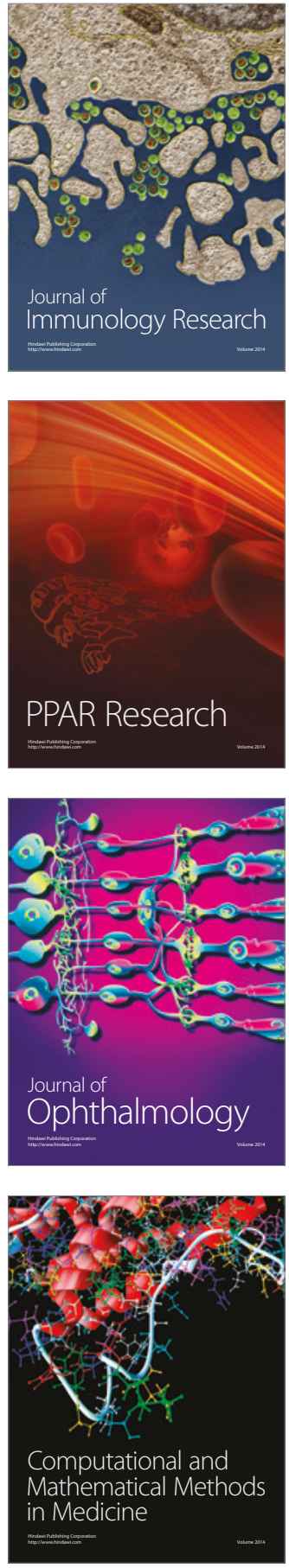

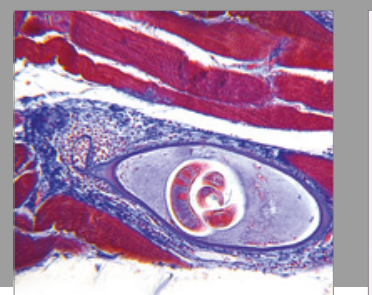

Gastroenterology Research and Practice
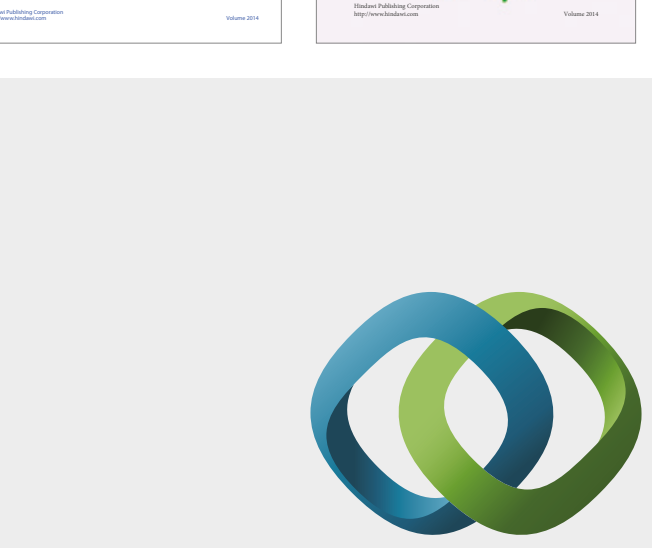

\section{Hindawi}

Submit your manuscripts at

https://www.hindawi.com
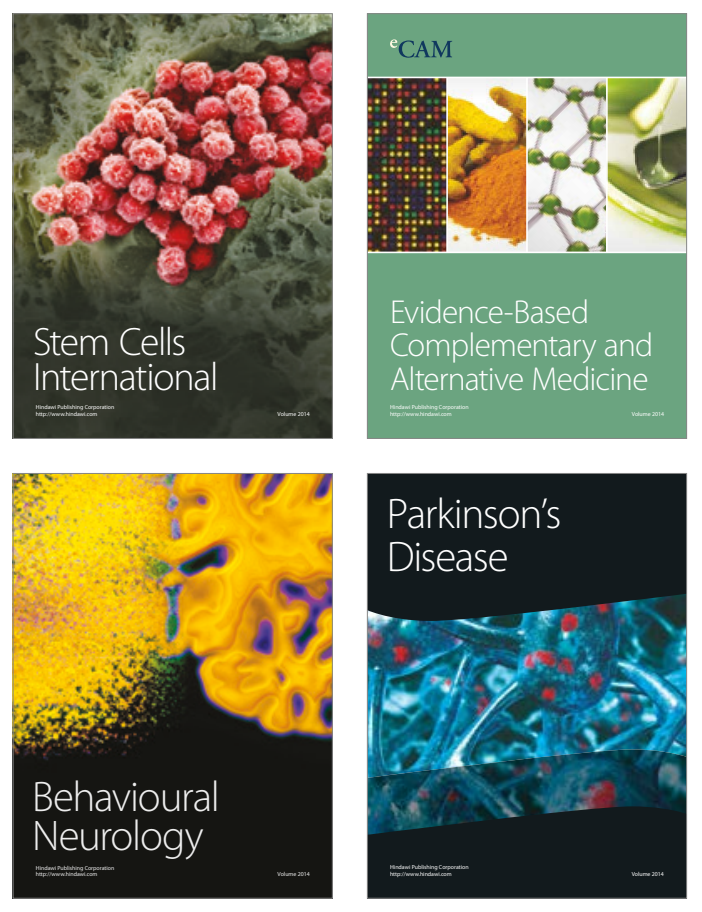
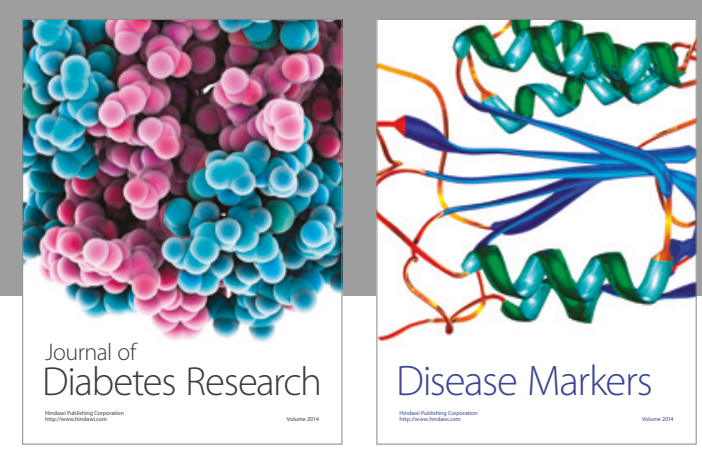

Disease Markers
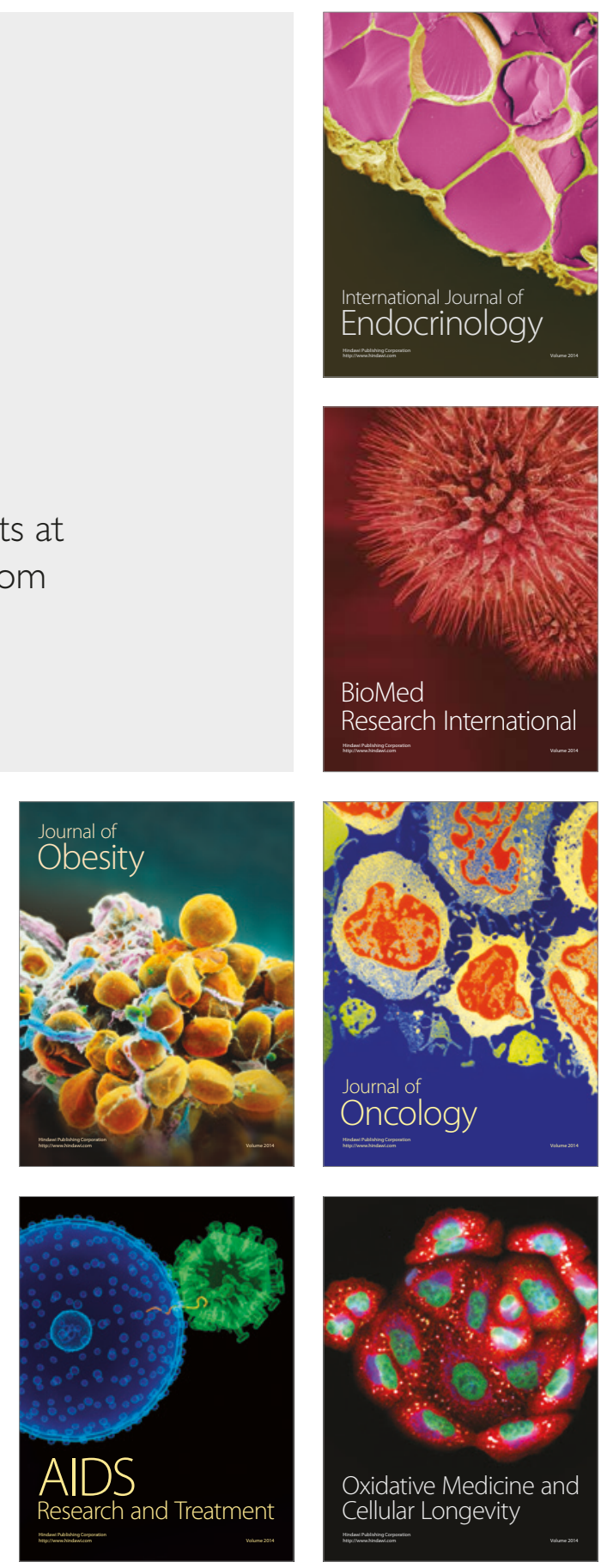